\title{
Large Eddy Simulation of a Turbulent Flow in a
} Differentially Heated Cavity with Rayleigh Numbers up to $10^{11}$ : Part I. Numerical Methods

\author{
Djedid Taloub $^{1,2}$ and Abdelhadi Beghidja ${ }^{1,3}$ \\ 1. Laboratory of Renewable Energy and Sustainable Development (LRESD) Constantine1 University, Constantine 25000, Algeria \\ 2. Faculty of Science M'sila University, M'sila 28000, Algeria \\ 3. Mechanical Department of Engineering, Constantine1 University, Constantine 25000, Algeria
}

Received: June 14, 2013 / Accepted: June 20, 2013 / Published: August 25, 2013.

\begin{abstract}
Large Eddy Simulations of the anisothermal turbulent flows has been used in the context of interaction problems thermal fluid, structure. In this context, the aim of this work lies not only in identifying the various elements that may underestimate the temperature fluctuations at the interface fluid-solid but also the introduction of the models capable of reproducing the physical setting Thurs. At first, the choice of convection scheme <optimizing > the scalar transport has led to the adoption of an upwind scheme of theorem 2 order. The use of models with conventional walls showed weaknesses in the estimation of temperature fluctuations bet stalls in the case of boundary layers attached. This work presents a numerical validation of LES-WALE model using the results of the $K$-epsilon model, this study is based on 3D numerical simulation using FLUENT code calculates to determine the longitudinal velocity, the thermal fields for the configuration the and speed vectors $(\mathrm{U}, \mathrm{V}, \mathrm{W})$ for configuration in the plane of the recirculation zones for the case of different values of flow Rayleigh. Therefore, the results have good agreement with those of k-epsilon model, as they show the difference between the cases of flows.
\end{abstract}

Key words: Cavity, LES, turbulent, fluent, $K$-epsilon.

$\begin{array}{ll}\text { Nomenclature } \\ T: & \text { Local temperature } \\ T_{m}: & \text { Average temperature } \\ T_{h}: & \text { Hot wall temperature } \\ T c: & \text { Cold wall temperature } \\ x, y, z: & \text { Cartesian coordinates } \\ u, v, w: & \text { Components of velocity (not filtered } \\ P r: & \text { Prandtl number } \\ R a: & \text { Rayleigh number }\end{array}$

Greek Letters

$\mu$ : Dynamic viscosity

$\mu_{t}: \quad$ Turbulent viscosity

$\rho: \quad$ Density

$C_{p}: \quad$ Heat capacity with constant pressure

$\lambda$ : Thermal conductivity

Corresponding author: Djedid Taloub, assistant professor, research fields: mechanics of fluid, environment and energy, modeling and simulation. E-mail: djtaloub@yahoo.fr.

$\begin{array}{ll}v: & \text { Kinematic viscosity } \\ \alpha: & \text { Thermal diffusivity }\end{array}$
Abbreviations
DNS: Direct numerical simulation
LES: Large eddy simulation
CFD: Computational methods for fluid dynamic

\section{Introduction}

Natural convection in parallelepiped cavities with vertical walls and differentially heated constitutes a basis configuration of various industrial systems, and particularly a reference case very simple for the development and validation of numerical simulation of flows natural convection.

The study of the natural convection of fluids in the cavities has been a very large number of both theoretical as experimental work. The interest of this 
study reside in its involvement in many natural and industrial processes such as cooling of electronic circuits and nuclear reactors, building insulation, metallurgy, crystal growth for the semiconductor industry drivers, etc..

The fluid flow, whether laminar or turbulent, are described by the system of partial differential equations. Thus, all physical phenomena are governed by the system formed by the equations of continuity, moving amount and energy that must be resolved to know the characteristics of the temperature field and flow field. The main purpose of the numerical simulation is to determine the physical behavior of the system submitted to heat transfer and can be important and instationary.

Concerning the system composed of anisothermal turbulent fluid flow and solid structure, problems related to thermal fatigue involve important features, other than a medium of temperature fields, such as frequency spectral and amplitude of temperature fluctuations. These temperature fluctuations depend mainly of investigated physical configuration (which can be very complex), the regime of flow (i.e., Reynolds number), the Prandtl number and the nature of the thermal coupling between the fluid and the solid.

This work is based on many previous studies both experimental and numerical, including Mergui [1-2], Salat [3-4] Lankhorst [5], Tian [6] Ampofo [7] examined numerically flows turbulent natural convection in a parallelepiped cavity. Many numerical investigations have also been conducted with cavities of modest size or $R a_{h}<10^{9}$ [8-9], beyond $\left(R a_{H}>10^{10}\right)$.

We consider here a configuration of natural convection in a parallelepiped cavity fluid, where a temperature gradient is imposed between two vertical walls, and the other walls are assumed to adiabatic.

There are examples of applications such a configuration in the solar systems, double glazed windows, or the description of the air flow within a room.

The aim of this work is to compare the results of
Fluent code to those proposed references. We study particularly the isothermal and vertical velocity profile, the calculation is performed from the temperature at the wall.

\section{Modeling of the Problem}

The physical model considered is shown schematically in Fig. 1 which is a three-dimensional parallelepiped cavity of large size $(H=2.46 \mathrm{~m}$ high, $L$ $=0.385 \mathrm{~m}$ width, $P=0.72 \mathrm{~m}$ deep).

The fluid within the cavity is considered incompressible and Newtonian. Because studying heat transfer appears only by natural convection, radiation is not taken into consideration in the numerical model. The dissipation of heat by viscous friction is neglected. The Boussinesq approximation is considered. The fluid within the cavity is air.

For the boundary conditions of the walls, the two vertical walls are considered isothermal. $T_{c}$ is the temperature of the hot wall and the $T_{f}$ of the cold wall. Other surfaces delimiting the cavity are considered adiabatic, therefore null heat flux.

The gravitational acceleration $g$ is taken into consideration. The study is performed for different Rayleigh numbers obtained by variation of the temperature difference between the hot and cold walls. $T_{c}$, hot wall; $T_{f}$, cold wall; $L_{D}$, right side wall; $L_{G}$, left side wall; $P_{d}$, ceiling and $P_{c}$, floor.

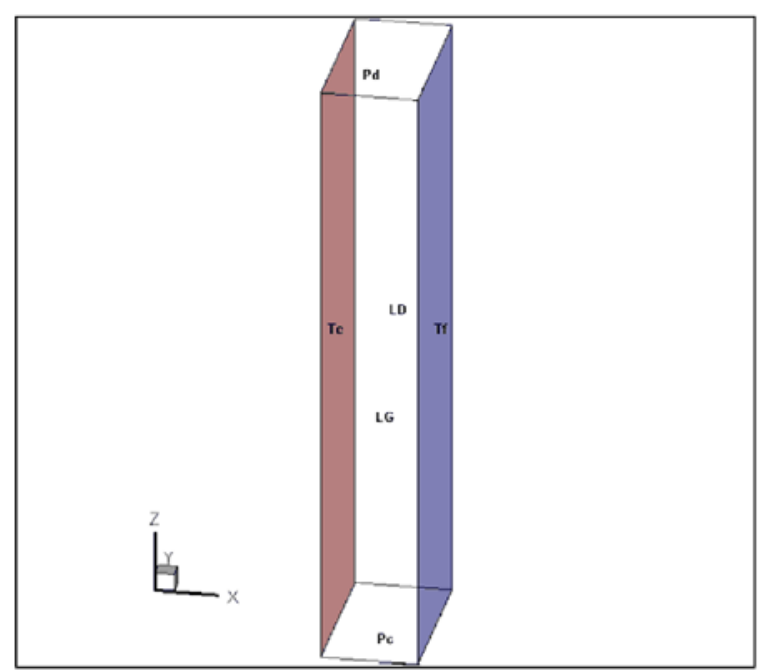

Fig. 1 Geometric configuration of the 3D model. 


\subsection{Boundary Conditions}

$$
\begin{aligned}
& x=0 \Rightarrow u=v=w=0, T=T C \\
& x=L, y=P \Rightarrow u=v=w=0, T=T f \\
& y=0, y=P \Rightarrow u=v=w=0, \frac{\partial T}{\partial y}=0 \\
& z=0, z=H \Rightarrow u=v=w=0, \frac{\partial T}{\partial z}=0
\end{aligned}
$$

\section{Numerical Simulation of Turbulence}

Numerical simulation of turbulent and concepts related to the turbulence and that can provide adapted and effective modeling flows. We introduced the same equations governing our flows through this article and after posing problems of closing LES.

\subsection{Basic Equations}

The equations of evolution are used to describe the flow of an incompressible fluid in its movement. Firstly reflects the conservation of mass locally, the other is conservation of movement quantity, and the third equation takes to reflect the heat transfer in the case of isothermal flow: energy equation.

The forms of these equations are different depending on the assumptions about the type of flow and fluid considered. In this study, we assume an incompressible fluid with constant thermodynamic properties ( $\mu=$ const, $\rho=$ constant, $\lambda=$ constant, $C p=$ constant).

By applying a low-pass filter to results equations and the above assumptions, the system of equations to be solved in a filtered LES approach [10] we obtain:

$$
\begin{gathered}
\frac{\partial \overline{u_{i}}}{\partial x_{i}}=0 \\
\frac{\partial \overline{u_{i}}}{\partial t}+\frac{\partial}{\partial x_{j}}\left(\overline{u_{j}} u_{i}\right)=-\frac{1}{\rho} \frac{\partial \bar{p}}{\partial x_{i}}+\frac{\partial}{\partial x_{j}}\left(2 \overline{S_{i j}}+\overline{\tau_{i j}}\right)(2) \\
\frac{\partial \bar{T}}{\partial t}+\frac{\partial}{\partial x_{j}}\left(\overline{u_{i}} \bar{T}\right)=\frac{\partial}{\partial x_{j}}\left(\alpha_{f} \frac{\partial \bar{T}}{\partial x_{j}}+\overline{\Theta_{i}}\right)
\end{gathered}
$$

$\overline{S_{i j}}$ : Corresponds to the rate tensor of resolved deformation given by:

$$
\overline{S_{i j}}=\frac{1}{2}\left(\frac{\partial \overline{u_{i}}}{\partial x_{j}}+\frac{\partial \overline{u_{j}}}{\partial x_{i}}\right)
$$

The new terms $\overline{\tau_{i j}}$ and $\overline{\Theta_{i}}$ from the filter respectively represent the tensor sub-mesh stress (or Reynolds stress) and heat flux sub-mesh and are defined by:

$$
\begin{gathered}
\overline{\tau_{i j}}=\overline{u_{i} u_{j}}-\overline{u_{i} u_{j}} \\
\overline{\Theta_{i}}=\bar{T} \overline{u_{i}}-\overline{T u_{i}}
\end{gathered}
$$

Modeling constraints sub-mesh $\tau_{i j}$ is based on an assumption of sub-mesh viscosity (Boussinesq hypothesis) linking constraints in the mesh deformation rate tensor resolved $S_{i j} \quad$ [10]:

$$
\overline{\tau_{i j}}-\frac{1}{3} \delta_{i j} \overline{\tau_{k k}}=2 v_{t} \overline{S_{i j}}
$$

Eq. (2) can be written as:

$$
\frac{\partial \overline{u_{i}}}{\partial t}+\frac{\partial}{\partial x_{j}}\left(\overline{u_{j} u_{i}}\right)=-\frac{1}{\rho} \frac{\partial \bar{p}}{\partial x_{i}}+\frac{\partial}{\partial x_{j}}\left(2\left(v+v_{t}\right) \overline{S_{i j}}\right)(8)
$$

The Smagorinsky model is based on an assumption of mixing length in which it is considered that the viscosity is proportional to the mesh length scale (here associated with the filtering equations, namely the characteristic mesh size) denoted $\Delta$ and a velocity scale determined by the product $\Delta\|\bar{S}\|$, where $\|\bar{S}\|$ is the norm of the strain rate defined tensor and resolved by Ref. [10]:

$$
\|\bar{S}\|=\sqrt{2 \overline{S_{i j} \overline{S_{i j}}}}
$$

Finally, writing the Smagorinsky model is as follows:

$$
v_{t}=\left(C_{S}\right)^{2}\|\bar{S}\|
$$

The constant $\mathrm{C}_{\mathrm{s}}$ is determined from the assumption of local equilibrium between production and dissipation of turbulent kinetic energy.

\subsection{Numerical Schemes}

We use the following calculation:

- The power-law schema: this one is best placed to capture the physical phenomena of heat transfer;

- The discretization scheme of pressure velocity 
coupling is SIMPLE;

- The convergence criterion used is the under-relaxation.

\subsection{Mesh}

One of the asked questions is what kind of Mesh (regular or not tightened to the walls) used for different Rayleigh numbers and different configurations considered. This, because we consider the results as satisfactory as from the moment no further refine the space influences more significantly.

We initially tried to use a mesh with no regular space, but the results are not very satisfactory. In addition, the knowledge of boundary layer phenomena, it became clear that it was preferable to use a finer mesh to the walls. This type of mesh has been used for the rest of the study.

\subsection{Validation}

In order to verify the accuracy of the numerical results obtained in the present work, a validation of the numerical code was made taking into account some numerical studies available in the literature. Ampofo results [11] obtained in the case of a square cavity containing air, were used to test our simulation by Fluent. The comparison was made by considering the Rayleigh number $1.58 \times 10^{9}$. Comparison of velocity profiles along the plane medium shows excellent agreement.

\section{Results and Discussion}

The natural convection was measured in an air-filled cavity, where the dimensions are: $H=$ $6,3896 L$ in $x, y$ and $z$ directions.

Isothermal vertical walls with a temperature difference of different $\Delta T$.

The accord between the DNS [12] and the LES model looks fairly good for the thermal field Fig. 3.

The obtained profiles (Fig. 5 of velocity profiles and temperature with the LES model, DNS and experience) show the ascending flow in adjacent to the hot wall. The amplitude of the DNS in the layer near the wall and in the center of the cavity. Maximum amplitude issue from the experiment, for a Rayleigh number higher than $10^{6}$ are slightly important.

Betts and Daffa'alla [13, 14] indeed indicate the existence of a secondary flow directed from the cold to the hot wall.

For the extension of the studied cavity, the intensity of convection differs depending on the value of the Rayleigh number. Indeed, more $R a$ increases, the convection is growing. This is explained in Fig. 3. by the longitudinal section $z=1.23$ of the air gap, showing the different boundary layer flow.

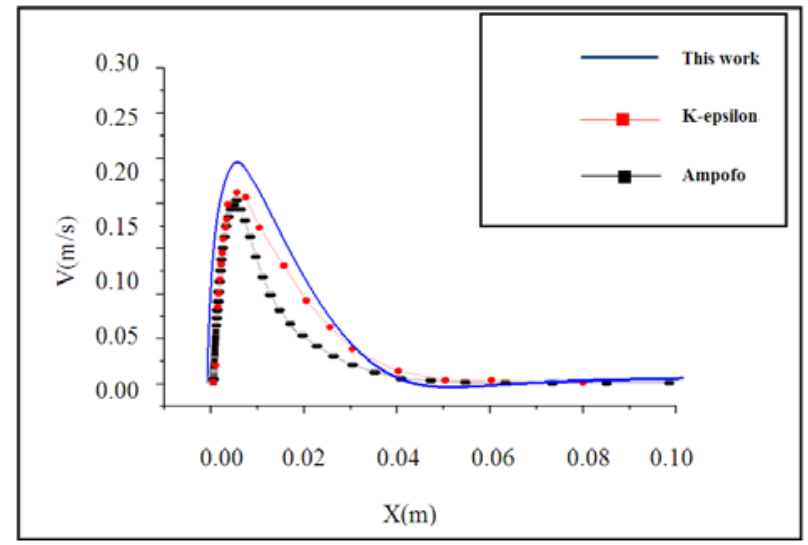

Fig. 2 Comparison of velocity profile $V$ along the $y=0.36$ m.

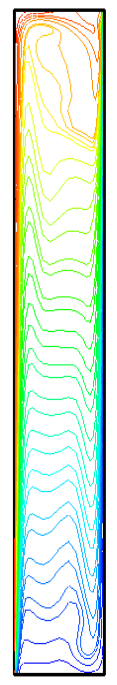

(a)

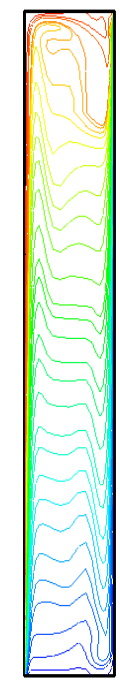

(b)

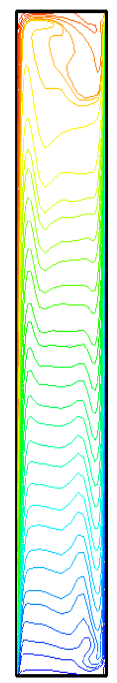

(c)

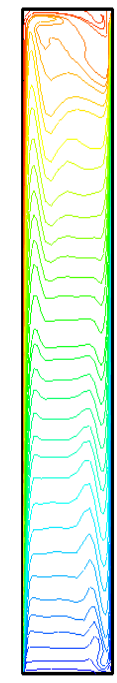

(d)

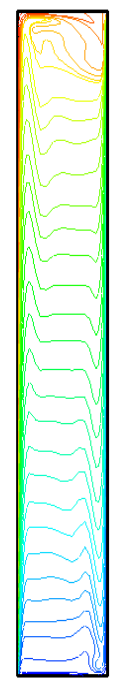

(e)
Fig. 3 Thermal fields for the configuration in the plane $y=$ $D / 2=0.36$ m, where: (a) $R a=2.5 \times 10^{9}$, (b) $R a=6.8 \times 10^{9}$, (c) $R a=2.5 \times 10^{10}$, (d) $R a=6.8 \times 10^{10}$ and (e) $R a=10^{11}$. 


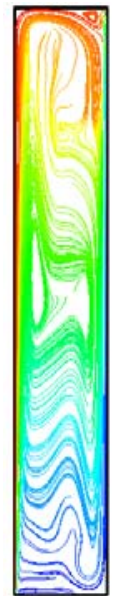

(a)

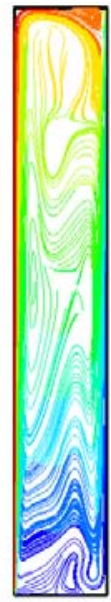

(b)

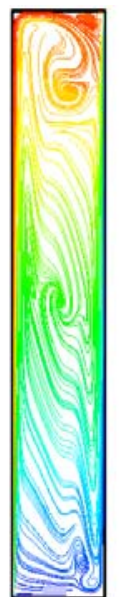

(c)

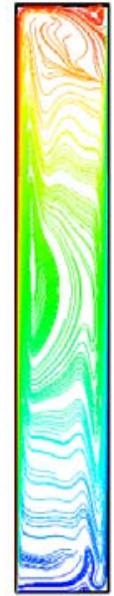

(d)

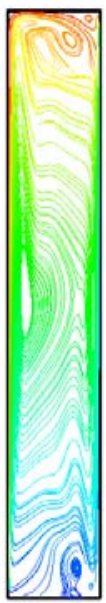

(e)
Fig. 4 Speed vectors $(U, V, W)$ for configuration in the plane $y=D / 2=0.36 \mathrm{~m}$ where: (a) $R a=2.5 \times 10^{9}$,(b) $R a=$ $6.8 \times 10^{9}$, (c) $R a=2.5 \times 10^{10}$, (d) $R a=6.8 \times 10^{10}$ and (e) $R a=$ $10^{11}$.

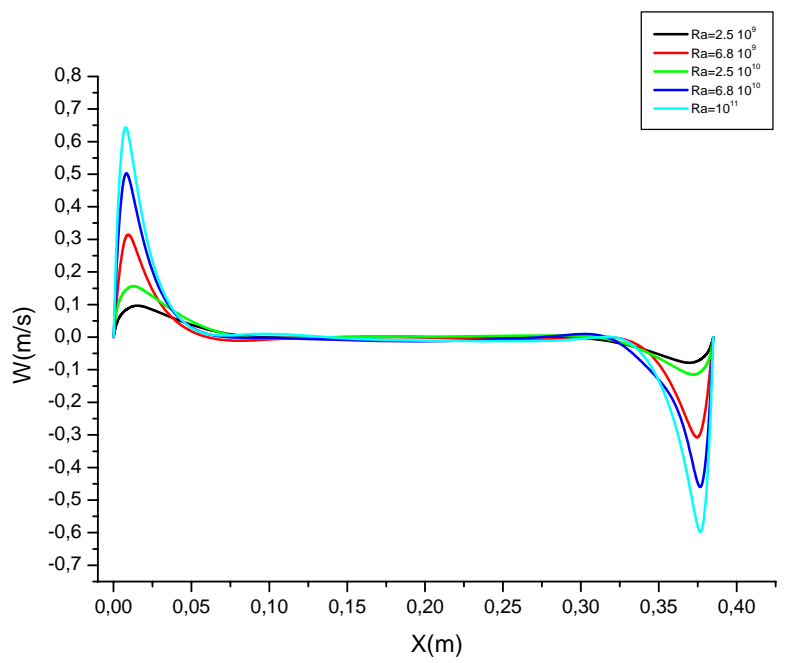

Fig. 5 Profile of vertical velocity for different values of the Rayleigh number in the configuration with the plane $y=$ $D / 2=0.36 \mathrm{~m}$ and $z=H / 2=1.23 \mathrm{~m}$.

Therefore the increase of Rayleigh correspondents to the increase of the natural convection. So we will fix the Prandtl number $P r=0.71$.

Furthermore, the velocity profile shown in Fig. 2 for a mid-sectional height $(H / 2)$ extension of the cavity $6.3896 L$ and $0.72 \mathrm{~m}$ in thickness is used to observe the enhancement of the convective in the cavity with increasing in imposed temperature variation.

The results also show the velocity distribution along the gap between active walls, the three distributions represent the three types of flow, namely that the regime
Table 1 Varying the Rayleigh number $(R a)$ based on the temperature of the gradient $(\Delta T)$.

\begin{tabular}{llllll}
\hline $\boldsymbol{\Delta} \boldsymbol{T}\left({ }^{\circ} \mathbf{C}\right)$ & 2 & 5 & 20 & 50 & 80 \\
\hline $\boldsymbol{R a}$ & $2.5 \times 10^{9}$ & $6.8 \times 10^{9}$ & $2.5 \times 10^{10}$ & $6.8 \times 10^{10}$ & $10^{11}$ \\
\hline
\end{tabular}

of conduction, transition and boundary layer. It may be noted that the maximum value of the vertical velocity increases with the augmentation of the Rayleigh number. The vertical velocity increasing proportional to the increase of the Rayleigh numbers that is to say the increase of $\Delta T$.

\section{Conclusions}

The flow of turbulent natural convection appears frequently in industrial applications, but relatively few studies have been conducted to describe it. Recently some Direct Numerical Simulation "DNS" were performed on simple configurations with control parameters $(R a)$ having moderate values. Thus, the experience is confronted with technical difficulties in measuring high-order correlations but especially in the boundary layer near the wall (inside layer) where the flow is completely different compared to the forced convection and again less well known.

We note that the flow has a parallel appearance and thermal stratification pronounced inside the cavity and the major part of the flow moves at sidewalls, this reflected by the formation of a boundary layer to the proximity of these walls. This structure is accompanied by recirculation zones in the lower corner and the upper right corner of the cavity.

We can confirm through this study the influence of the Rayleigh number so $\Delta T$ (since fluid characteristics rest unchanged), the convection and hence the trigger zone of turbulent instability.

Given the consistency of our results with those established experimentally and numerically by other, we were able to validate the calculation for the studied configuration by LES model and finite volume. Therefore, we have already addressed this problem with the simulation method (k- $\varepsilon$ strandart) [15-17].

The numerical study in the CFD code Fluent has enabled us to both validate the numerical simulations 


\section{Large Eddy Simulation of a Turbulent Flow in a Differentially Heated Cavity with Rayleigh Numbers up to 529 $10^{11}$ : Part I. Numerical Methods}

by comparing the results to those obtained by the correlations used in the literature and secondly to define a convective heat transfer coefficient through the Nusselt number for some study conditions that (dimensions) were not studied in the literature that will be presented and discussed in Part II of this work.

In conclusion, the results obtained are in good agreement with both experimental and numerical results.

\section{References}

[1] S. Mergui, F. Penot, Analyzes speeds and temperature of the air in natural convection in a cavity differentially heated with $\mathrm{Ra}=1.6910^{9}$, Int. J. Heat Mass Transfer 40 (14) (1991) 3427-3441.

[2] S. Mergui, Experimental characterization of the flows of air of natural and mixed convection in a closed cavity, Thesis Doctorate, University of Poitiers, 1993.

[3] J. Salat, F. Penot, Experimental approach of the natural convection of turbulent transition in a cubic cavity differentially heated VI Inter-University conference Frensh-Quebec Thermics of the Systems, (26-28 mai 2003, Quebec).

[4] J. Salat, Contribution to the study of the three-dimensional natural convection in cavity differentially heated. Thesis of Doctorate of the Poitiers University, 2004.

[5] A.M. Lankhorst, D. Angirasa, C.J. Hoogendoorn, LDV measurements of buoyancy induced flows in an enclosure at high Rayleigh numbers, Exp. Thermal and Fluid Science 6 (1993) 74-79.

[6] Y.S. Tian, T.G. Karayiannis, Low turbulence natural convection in an air filled square cavity: Part 1 . The Thermal and Fluid Flow Fields, IJHMT 43 (2000) 849-866.

[7] F. Ampofo, T.G. Karayiannis, Experimental benchmark data for turbulent natural convection in an air filled square cavity, IJHMT 46 (19) (2003) 3551-3572.
[8] G. De Vahl Davis, Laminar natural convection in an enclosed rectangular cavity, In. J. Heat and Mass Transfer 11 (1968) 675-1693.

[9] S. Xin, P. Le Quéré, Direct numerical simulation of two dimensional chaotic natural convection in a differentially heated cavity of aspect ratio 4, J. Fluid Mech. 304 (1995) 87-118.

[10] A. Chatelan, Larg eddy Simulation of the turbulent flows with heat transfers, Doctorate Thesis, Laboratory of Modeling and Development of Software (CEA Grenoble).

[11] F. Ampofo, Turbulent natural convection of air in a non-partitioned or partitioned cavity with differentially heated vertical and conducting horizontal walls, Exp. Thermal and Fluid Science 29 (2005) 137-157.

[12] F.X. Trias, A. Gorobets, M. Soria, A. OlivaD. Direct numerical simulation of a differentially heated cavity of aspect ratio 4 with Rayleigh numbers up to $10^{11}$ : Part I. Numerical methods and time-averaged flow, Int. J. Heat and Mass Transfer 53 (2010) 665-673.

[13] P.L. Betts, A.A. Dafa'Alla, Turbulent buoyant air flow in a tall rectangular cavity, UMIST?, Manchester, England.

[14] A. Dafa'Alla, P. Betts, Experimental stady of turbulent natural convection in a tall air cavity, Exp. Heat Transfer 9 (1996) 165-194.

[15] A. Beghidja, Dj. Taloub, Effect of Rayleigh number on the turbulent structures with boundary layer in a differentially heated, In. J. Mechanics and Energy (IJME) 1 (2) 2013.

[16] A. Beghidja, M. Bouafia, J.J. Vullierme, F. Yguel, A. Zammit, Modeling of the exchanges convective on the front verticals of cavities to great numbers of Rayleigh, General Review of Thermics Tom 34 (405) (1995) 515-526.

[17] F. Ampofo, Turbulent natural convection of air in a non-partitioned or partitioned cavity with differentially heated vertical and conducting horizontal walls, Exp. Thermal and Fluid Science 29 (2005) 137-157. 